\title{
The Primary Reconstruction of Three-Dimensional(3D) Digital Model of Intracranial Aneurysm(IA) with Fenestration Based on Raw Data of Computed Tomography(CT) Imaging
}

\author{
Yijun LIU ${ }^{1}$, Rui $\mathrm{LI}^{1}$, Weijian CHEN ${ }^{1, \text { a }}$, Nengzhi XIA ${ }^{1}$, Pengcheng $\mathrm{YE}^{2}$, Yuxia \\ DUAN $^{1}$, Yunjun YANG $^{1}$, Meshram Manmeet Manohar ${ }^{3}$
${ }^{1}$ Department of Radiology, The First Affiliated Hospital, Wenzhou Medical University, Wenzhou, 325000, China
${ }^{2}$ Department of Stomatology, The First Affiliated Hospital, Wenzhou Medical University, Wenzhou, 325000, China

${ }^{3}$ Department of Orthopaedics, The First Affiliated Hospital, Wenzhou Medical University, Wenzhou, 325000, China

acCorresponding author: Weijian CHEN email: wyyycwj@163.com Keywords: Intracranial Aneurysm, Fenestration; 3D Digital Model, Reconstruction; Computed
Tomography

\begin{abstract}
The diagnostic value of fenestration itself is limited but it is quite different when fenestration is accompanied by IA. In recent years, the reconstruction model of IA has become the domestic and international hot topic for discussion, however, it is rare to see the research about reconstruction model of IA with fenestration. Based on reliable CT imaging technologies and optimized modeling methods, we successfully reconstruct the 3D digital model of IA with fenestration by applying Mimics software combined with Geomagic studio software. This model can provide a necessary condition for the 3D finite element reconstruction model and computational fluid dynamic analysis, open a new approach for relative clinical hemodynamic study and operative treatment.
\end{abstract}

\section{Introduction}

Intracranial Aneurysm(IA) refers to the focal abnormal enlarged cavity of the intracranial artery. The incidence of IA is about 1-3\%[1-3] and IA can occur at any age. The rupture of IA can lead to about $80 \%$ of subarachnoid hemorrhage( $\mathrm{SAH})$. "Digital Medicine" is a new inter-disciplinary concept wherein information technology is combined with life sciences. Obviously, digital revolution is an inevitable trend in radiology with the emergence and development of modern medical imaging technology. The present study suggests that the hemodynamic factor has become the domestic and international hot point in recent years which is closely related to the occurrence, development and outcome of IA. Hemodynamic research of IA is mainly realized by applying the method of computational fluid dynamics(CFD) in engineering science and to construct the accurate and effective three-dimensional(3D) finite element model is the most important step in the process[4]. The domestic and international research about 3D finite element model of IA is extremely popular, however, it is rare to see research about 3D finite element model of IA with fenestration. Fenestration of intracranial artery(FIA),also called the name of fenestrated cerebral artery, is a rare but well-known vascular variation[5-6]that begins with a common origin, splits into two distinct endothelium-lined channels, which then rejoin distally[7]. The size of the fenestration varies from approximately $1 \mathrm{~mm}$ to several centimeters in length. Based upon the morphology and size, FIA can be sorted into two types: slit-like shape and convex-lens-like shape. In general, the diagnostic value of FIA itself is limited but it is quite different when FIA is accompanied by IA. Although Digital Subtraction Angiography(DSA) is the golden standard of diagnosing intracranial vessel disease at present, Computed Tomographic Angiography(CTA) has been widely used in clinical practice for imaging the intracranial vessels and detecting IA, FIA or other vascular 
abnormalities. Therefore, the aim of our study is to reconstruct the 3D digital model of IA with fenestration based on raw data of brain helical CT, making a good foundation for further 3D finite element model and related hemodynamic study.

\section{Materials and Methods}

\subsection{Modeling Environment}

We reconstructed the 3D digital model of IA with fenestration by personal computer(PC) platform. PC system specifications: HP P7370 Intel（R ) Core(TM)2 Duo CPU 2. 00 GHz，2. 00 GB storage, Windows 7 system. Modeling Softwares: medical 3D image generating and editing software Mimics 10.0(Materialise Company, Belgium), reverse engineering software Geomagic studio 12.0(Raindrop Company, USA).

\subsection{Collection of raw data}

We selected one representative patient of anterior communicating artery aneurysm with fenestration at the First Affiliated Hospital of Wenzhou Medical University as the study object(Male patient, 48 years old, sudden unconsciousness for 5 hours, "SAH" as the clinical diagnosis, "anterior communicating artery aneurysm, anterior communicating artery fenestration, large amount of SAH, and small amount of hemorrhage in bilateral ventricles" as the CTA result). The raw data of this patient was acquired by a 64-channel multidetector CT scanner (Lightspeed VCT 64 General Electric Medical Systems, Milwaukee, WI, USA) with $0.625 \mathrm{~mm}$ collimation and $0.5 \mathrm{~mm}$ interval(a pitch of $0.984,0.5 \mathrm{~s}$ per rotation, $100 \mathrm{kV}, 500 \mathrm{~mA}$ ). The scanning range was from the inferior edge of foramen magnum to the parietal tuber and the scanning line was parallel to the skull base. Iodixanol-320 injection was injected via the antecubital vein with an automatic power injector(Medrad Stellant D CT Injector, Warrendale, USA) at $4.0 \mathrm{ml} / \mathrm{s}$, with the use of Smart Prep and Sure Start at the common carotid artery for 64-channel scanner. The raw data of this patient was stored by Digital Imaging and Communications in Medicine(DICOM) standard format.

\subsection{Digital model reconstruction of IA with fenestration}

At first,we applied Mimics software to read consistent CT raw data of DICOM format directly and mark correct orientation of the image. The image threshold was adjusted carefully in order to show the vessel image as clearly as possible. We dealt with the image by the method of threshold segmentation combined with manual segmentation to obtain the most important region of interest(ROI) mostly including the region of IA, fenestration and parental artery(Figure 1-2), meanwhile, we removed surrounding tiny branches and interfering bony structures carefully. After that we used the 3D calculate function to reconstruct the model by choosing the 3D reconstruction button and it was stored and sent out by STL format. We then applied Geomagic studio software to read the 3D reconstruction model of STL format directly and make it meshed by triangle shape with deleting the unnecessary part. We chose the edit polygon tool to further optimize the model by adjusting the distribution of surface meshes with the special flip, split and shrink function,especially for the region of fenestration. Almost every triangle patch should be kept as the equilateral or equicrural shape. For optimizing the section of vessel wall better in order to reflect the real nature of blood vessel, we creatively utilized a smart method called the "Shell Method"(Figure 3). in the first step, we optimized sectional triangle patches one cycle around the region 0.5 centimeter distance toward the end of vessel wall. in the second step, we filled in the sectional region by the fill tool function. We finally completed all the step by shelling the same diameter for recovering the real nature of blood vessel better which is of great significance for further hemodynamic quantitative analysis. We tried our best to keep almost every triangle patch with symmetric angle and appropriate side length for the optimized 3D digital mode. In the end, we invited two experienced biomechanic experts to evaluate the final 3D digital model respectively. When two observers had different opinions, a discussion was held to reach the same consensus. The model was eventually stored and sent out by STL format after our agreement. 


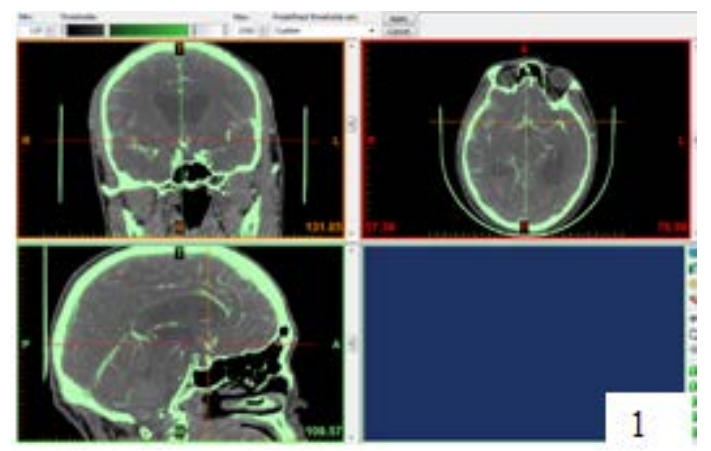

Fig.1 Set up the certain threshold by the method of automatic segmentation and choose custom type as the predefined thresholds set.

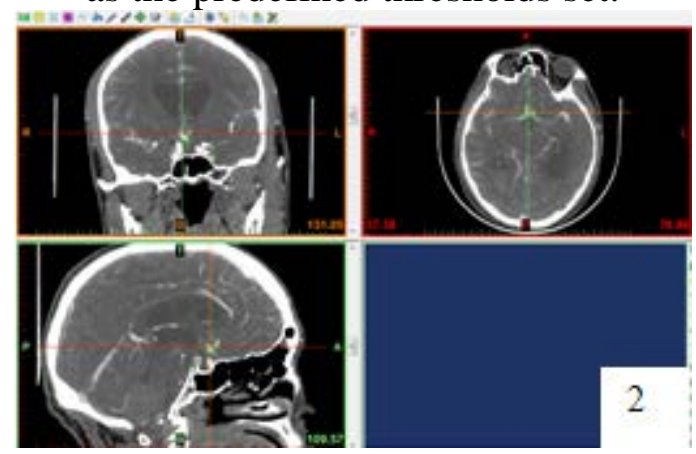

Fig.2 By the method of manual segmentation deal with the image to obtain the most important region of interest(ROI).

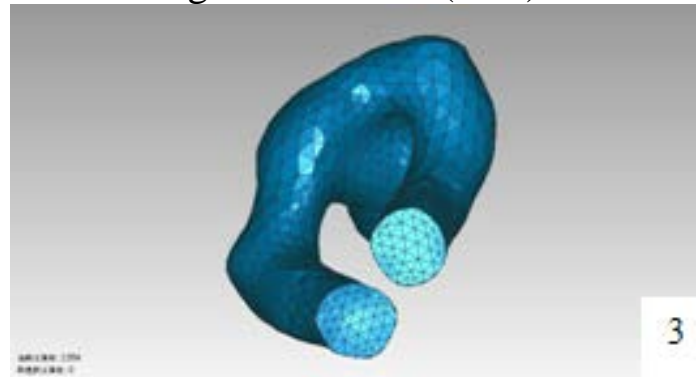

Fig.3 By the "Shell Method" reconstruct the 3D digital model of intracranial aneurysm with fenestration from the special view to show the vessel wall clearly.

\section{Results}

To the best of our knowledge, we successfully reconstructed the 3D digital model of IA with fenestration based on raw data of brain helical CT at the first time by applying Mimics software combined with Geomagic studio software to make the image recognition, reconstruction and optimization more accurate and effective(Figure 4-5).

The finished 3D digital model of IA with fenestration had high degree of digitalization, powerful practicality, great biological shape and structural similarity so that it was of great significance like a bridge connecting the subject of medical radiology and biomechanics. The quality of this model was relatively excellent because we not only reproduced the region of IA, fenestration and parental artery smoothly and completely, but also rotated and scaled the model randomly to make the relationship between them more visualized(Figure 6-7). In addition, it was possible for this model to be read into finite element and CFD softwares based on finite element methods to further calculate and analyze hemodynamic information of IA with fenestration, such as evaluating hemodynamic characteristics, simulating the flow of blood numerically in vitro to obtain related hemodynamic parameters(blood flow, pressure, shear stress and so on), thus explaining the forming reason of IA and even predicting the possibility of IA occurrence, development and rupture in FIA patient. 


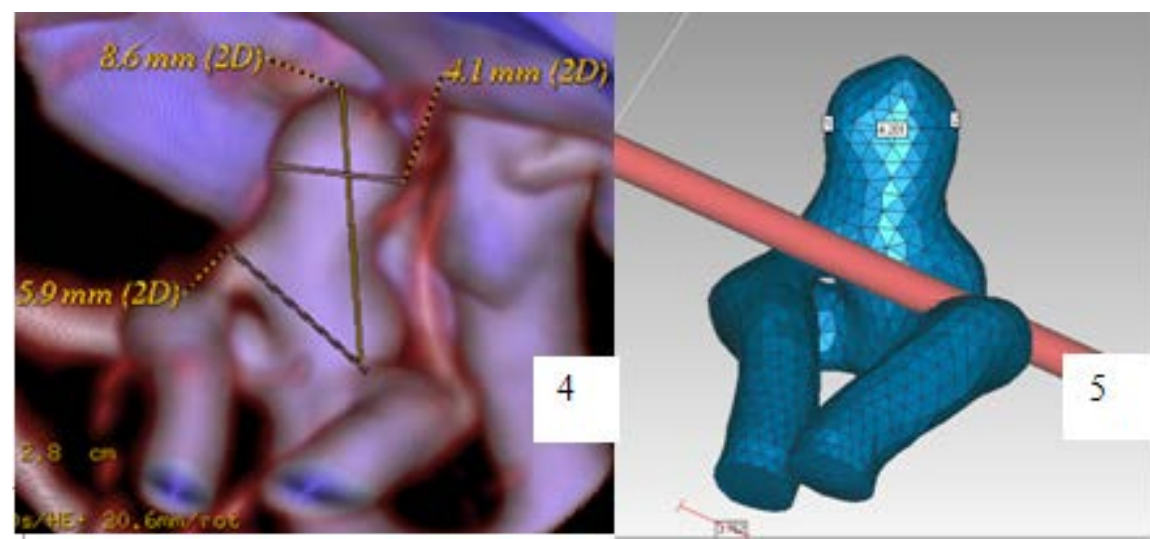

Fig.4-5 Male patient, 48 years old, sudden unconsciousness for 5 hours, "SAH" as the clinical diagnosis. Figure.4 for the VR image of CTA reconstruction, shows anterior communicating artery aneurysm with fenestration. Figure.5 for the image of 3D digital reconstruction model by applying Mimics software combined with Geomagic studio software based on the CT raw data of fig.4.

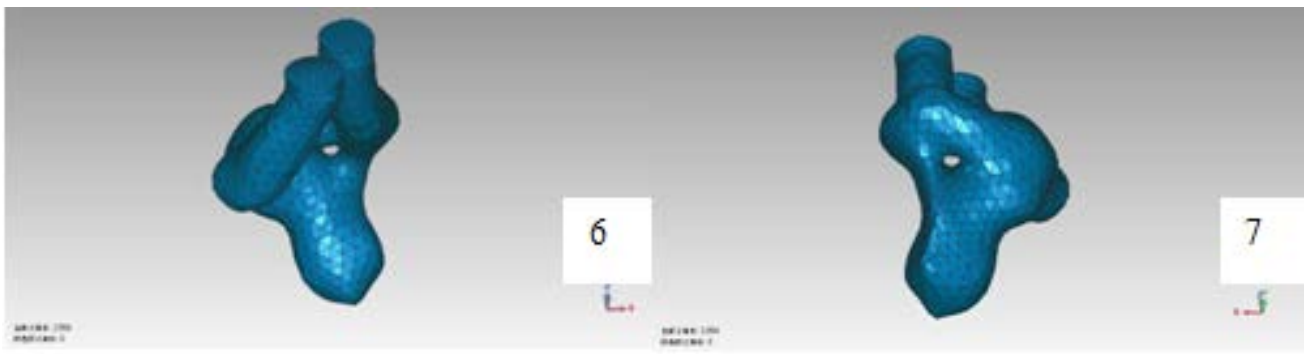

Fig.6-7 Figure 6 for the 3D digital model of intracranial aneurysm with fenestration from the top view. Figure 7 for the 3D digital model of intracranial aneurysm with fenestration from the bottom view.

\section{Discussion}

As we know, aneurysm rupture is the most common cause of non-traumatic SAH. In the event of the patient with rupture and hemorrhage forming large hematoma, it carries a high disability and mortality rate affecting quality of life severely. In this case, we found small amount of hemorrhage in bilateral ventricles meaning that the extent of $\mathrm{SAH}$ was severe and hence this case was characteristic. About $90 \%$ of IA originates from internal carotid artery(ICA) system which is mostly connected to the parental artery by aneurysm peduncle(or called aneurysm neck) including 30-35\% of IA from anterior communicating artery(Acoa) region, this case was consistent with the situation. The occurrence of FIA, associated with original embryonic vascular dysplasia, is often seen in Acoa region followed by vertebrobasilar system and middle cerebral artery region[8]. According to microsurgical research, the incidence of Acoa fenestration is about 40\%[9]. In this case, fenestration was located at Acoa, Acoa fenestration is related to the embryonic initial vascular network anastamosis and the hemodynamic condition between the Anterior Cerebral Artery(ACA) and Middle Cerebral Artery(MCA), etc[10]. According to the standard classification by Uchino A et al[11], it was a slit-like fenestration because the fenestrated vessel was small and vascular space was not obvious.

With the fast development of imaging technologies, imaging reconstruction of IA has already been realized, however, neither hemodynamic quantitative study nor real hemodynamic information has been done with these methods. In fact, current researches have changed to pay attention to construct the 3D finite element simulative model based on imaging data and analyze hemodynamic information of IA thus knowing hemodynamic mechanism of IA better. some authors started to focus on the 3D reconstruction model based on common clinical imaging data long ago[12-14]. In 2008, Wang et al[15] relied on CT raw data of an IA patient to establish a 3D finite element model by the Mimics software and the ANSYS software providing a chance for further hemodynamic 
study. In 2010, Liu et al[16] established finite element models by the Mimics software and the ANSYS software through 3D-DSA volume data of ten IA patients. Eventually, coincidence rate was $100 \%$ and all models were available for hemodynamic calculation and analysis of IA.

FIA is often accompanied by IA, FIA is associated with the formation of IA in most articles[17-18], however, there are a few articles demonstrating that there is no definite relationship between FIA and IA[8]. The structural feature of FIA is that the vessel part can be divided into two separated endothelium-lined channels and there are partial medial defects at the proximal and distal bifurcation of fenestration[19]. One hypothesis is that the vortex flow induces medial defects of partial vessel wall at the proximal and distal part of fenestration leading to the occurrence of IA[10]. According to the relationship between FIA and IA anatomy, IA was adjacent to FIA but not within the fenestration for this case. Some scholars indicated that FIA of this type had obvious vortex flow within the vessel making endothelium become obviously thinner so that it increased the chance of IA distinctly. Furthermore, other studies showed that FIA patient had high incidence of IA because of medial defect of vessel wall with high hemodynamic pressure so we inferred that the occurrence of Acoa aneurysm should be associated with that for this case. Although FIA is mostly discovered by chance at autopsy or angiography which has no certain clinical symptom[10], it is of clinical significance when accompanied by other intracranial vessel abnormalities such as saccular aneurysm and arteriovenous malformation as well as associated with the treatment effect of vascular interventional method and neurosurgical operative approach. Therefore, we made it possible to do hemodynamic individual research by reconstructing the 3D digital model of IA with fenestration in order to evaluate disease risk and make therapeutic plan thus providing reliable evidence for clinical operative treatment. Although a former article was published to analyze hemodynamic simulative values of IA with fenestration, our study subject(CT data of Acoa aneurysm with fenestration) was absolutely different from this one(DSA data of vertebral basilar artery fenestration with aneurysm).

In brief, CTA is a kind of minimally invasive angiography especially for the diagnosis of vascular disease. CTA is helpful for observing blood supply of all intracranial arteries and obtaining clear and fine 3D vessel images. Because reconstructed images are not influenced by intracranial hemorrhage, CTA is especially suitable for emergency patients, therefore, CTA can replace invasive DSA to some extent. Some studies showed that the sensitivity and specificity of CTA is higher than DSA for finding intracranial aneurysms at different sizes and locations[20]. Through the large sample research, Sanders et al drew a conclusion that the prevalence of FIA by DSA was only $0.7 \%$, much lower than the prevalence of FIA by CTA[8].

At present, Mimics software is the most common medical 3D image generating and editing software which has powerful function for constructing models on the base of cross-sectional images. As we suggest, the recognition and reconstruction for CT raw data by Mimics software is just like " manual CTA reconstruction " in reality. Now it is essential to apply the maximum intensity projection(MIP) method and volume rendering(VR) method to do the CTA reconstruction of intracranial vessels. The MIP image is helpful for showing intravascular and extravascular condition and has advantages for observing vessel walls and continuous distribution of vessels, however, the quality of MIP image is relatively poor. The VR image is helpful for dimensional relationship between vessels and surrounding important structures, moreover, the VR image has higher resolution, image quality and more colorful presentation compared with the MIP image. In contrast, intravascular detailed information is not available by the VR method. To our satisfaction, we apply Mimics software to fulfill demands of both MIP method and IA method for the 3D reconstruction. Mimics software has powerful operability so that we can take accurate and careful measures at any part of images by setting the certain threshold. We dealt with the image by the method of threshold segmentation combined with manual segmentation during the process, meanwhile, we removed surrounding tiny branches and interfering bones carefully in order to do exact and direct operation about the most important ROI and ensure reliability and validity of the reconstructed model to largest extent. In addition, we applied Mimics software to read CT images of DICOM format directly. It was convenient and reliable so that we did not need to make image conversion in any 
form during the preparation and avoid making errors caused by personal factors.

At present, Geomagic studio software is a very representative reverse engineering ( RE) software which has powerful function for optimizing 3D reconstructed models. So-called "reverse" is relative to "forward". Forward process is the step from designing papers to making real products, however, $\mathrm{RE}$ process is the step from getting corresponding point cloud data by making real products, sample pieces and scanned images as the study object to creating computer aided design(CAD) curved models. RE can be divided into three modules: collecting point cloud data; processing data, which is very important and is complicated because of the need to fetch characteristic points, lines and surfaces as well as reconstruct and smoothen scattered data points; and reconstructing the curved model. CAD modeling method of RE is aimed to reconstruct the geometrical shape so that the reconstructed model can meet the demand of accuracy and smoothness. According to different curved topological forms, the curved reconstructed method can be divided into two types: triangle patch and rectangle patch. In this case, we applied the method of triangle patch and took effective optimized strategies to optimize surface meshes for great effect which is better than directly calling Remesh function of FEA module by Mimics software. As we know, there are two different physiological pressures formed by the impact of blood flow to the vessel wall: one is the impact stress from inertial force of blood flow vertical to the vessel wall and the other one is the wall shear stress(WSS) which is the viscous friction from blood flow parallel to the vessel wall. Regional increase of WSS can dilate or even degenerate part of the vessel walls thus leading to the formation or enlargement of aneurysms[12]. In this case, we paid much attention for optimizing the section of vessel wall, because whether the optimized model is successful or not depends not only on the optimization of surface meshes, but also the real nature of vessel wall shape. Therefore, we utilized the Shell Method to recover the current nature of blood vessel which is of great benefit for reflecting the real blood flow condition. In a sense, we solved the problem that the shape of terminal vessels reconstructed by Mimics software lacked reality and have reason to believe that it would bring us inspiration for overcoming such shortcomings. In addition, we applied Geomagic studio software to read the 3D reconstruction model of STL format directly and delete the inevitable unnecessary part by Mimics software in order to make it more convenient and efficient.

As far as we are concerned, the 3D digital model reconstructed by Mimics software combined with Geomagic studio software is mainly applied to the field of bone and joint system[21-22]. After considering radiologic characteristics and clinical values of IA with fenestration adequately, we successfully reconstructed the 3D digital model of IA with fenestration by applying Mimics software combined with Geomagic studio software based on reliable CT imaging technologies and optimized modeling methods, providing a necessary condition for the 3D finite element reconstruction model and CFD analysis, opening a new approach for relative clinical hemodynamic study and operative treatment.

\section{Acknowledgement}

This study was supported, in part, by the National Key Project of Scientific and Technical Supporting Program funded by the Ministry of Science and Technology of China during the 12th Five-Year Plan (Project No. 2011-BAI08B09). The Wenzhou Municipal Science and Technology Project (Y20140029).

\section{References}

[1] Rinkel GJ. Natural history, epidemiology and screening of unruptured intracranial aneurysms. [J] . Rev Neurol (Paris). 2008; 164: 781-786.

[2] Weir B. Unruptured intracranial aneurysms: a review. [R] . J Neurosurg. 2002; 96: 3-42.

[3] Vlak MH, Algra A, Brandenburg R, Rinkel GJ. Prevalence of unruptured intracranial aneurysms, with emphasis on sex, age, comorbidity, country, and time period: a systematic review and meta-analysis. [J] . Lancet neurology. 2011; 10: 626-636. 
[4] Steinman DA, Milner JS, Norley CJ, et al. Image-based computational simulation of flow dynamics in a giant intracranial aneurysm. [J] . Am J Neuroradiol. 2003; 24: 559-566.

[5] Dey M, Awad IA. Fenestration of supraclinoid internal carotid artery and associated aneurysm:embryogenesis,recognition,and management. [J] . WorldNeurosurg. 2011; 76(6): 592-595.

[6] Bentura JE, Figueiredo EG, De Monaco BA, et al. Vertebrobasilar artery junction aneurysm associated with fenestration. [J] . ArqNeuropsiquiatr. 2010; 68(2): 312-314.

[7] Bayrak AH, Senturk S, Akay HO, Ozmen CA, Bukte Y, Nazaroglu H. The frequency of intracranial arterial fenestrations: a study with 64-detector CT-angiography. [J] . Eur J Radiol 2011; 77: 392-396.

[8] Aditya B, Ritchard IA, Jeremy W, et al. Intracranial arterial fenestrations: frequency on CT angiography and association with other vascular lesions. [J] . Surg Radiol Anat. 2008; 30(5): 397-401.

[9] Gast AN, van Rooij WJ, Sluzewski M. Fenestrations of the anterior communicating artery: incidence on 3D angiography and relationship to aneurysms. [J] . AJNR. 2008; 29(2): 296-298.

[10]Sanders WP, Sorek PA, Mehta BA, et al. Fenestration of intracranial arteries with special attention to associated aneurysm and other anomalies. [J] . ANJR. 1993; 14(3): 675-680.

[11]Uchino A, Kato A, Takase Y, Kudo S. Basilar artery fenestrations detected by MR angiography. [J] . Radiat Med. 2001; 19: 71-74.

[12] Hassan T, Timofeev EV, Saito T, et al. Computational replicas: anatomic reconstructions of cerebral vessels as volume numerical grid sat three-dimensional angiography. [J] . AJNR Am J Neuroradiol. 2004; 25(8): 1356-1365.

[13]Cebral JR, Castro MA, Burgess JE, et al. Characterization of cerebral aneurysms for assessing risk of rupture by using patient-specific computational hemodynamics models. [J] . AJNR Am J Neuroradiol. 2005; 26(10): 2550-2559.

[14]Hassan T, Timofeev EV, Ezura M, et al. Method of 3D image-based computational fluid dynamics. [J] . AJNR Am J Neuroradiol. 2003; 24(6): 1075-1082.

[15]Fuyu Wang, Bainan Xu, Lei Liu, et al. A three-dimensional finite element model of intracranial aneurysm based on CT transverse image data. [J] . Chin J Neuromed. 2009; 8(6): 585—587.

[16]Lei Liu, Bainan Xu, Fuyu Wang, et al. A three-dimensional finite element model of intracranial aneurysm based on DSA image data. [J] . Chinese Journal of elderly cerebrovascular disease. 2010; 12(8): 687-689.

[17]Peluso JP, van Rooij WJ, Sluzewski M,et al. Aneurysms of the vertebrobasilar junction: incidence, clinical presentation, and outcome of endovascular treatment. [J] . AJNR. 2007; 28(9): 1747-1751.

[18]Sun ZK, Li M, Li MH, et al. Fenestrations accompanied by intracranial aneurysms assessed with magnetic resonance angiography. [J] . NeurolIndia. 2012; 60(1): 45-49.

[19]Hacein-Bey L, Muszynski CA, Varelas PN. Saccular aneurysm associated with posterior cerebral artery fenestration manifesting as a subarachnoid hemorrhage in a child. [J] . AJNR. 2002; 23: 1291-1294.

[20]Lu L, Zhang LJ, Colin S. Poon , et al. Digital subtraction CT angiography for detection of intracranial aneurysms : comparison with three-dimensional digital subtraction angiography. [J] . Radiology. 2012; 262(2): 605-612.

[21]Liacouras PC, Wayne JS. Computational modeling to predict mechanical function of joints: application to the lower leg with simulation of two cadaver studies.[J] . J Biomech Eng. 2007; 129(6): 811-817.

[22]Keyak JH, Meagher JM. Automated three-dimensional finite element modeling of bone:a new method.[J] . J Biomed Eng. 1990; 12(5): 389-397. 